\title{
Análise da mastigação e da saciedade em escolares
}

\section{Analysis of chewing and satiety in schoolchildren}

\author{
Bruna Vieira Santos ${ }^{1,}(\mathbb{D}$, Martha Elisa Ferreira de Almeida² (D) \\ 1. Nutricionista pela Universidade Federal de Viçosa (UFV), Rio Paranaíba, MG, Brasil. 2. Docente do Curso de Nutrição da Universidade Federal de Viçosa \\ (UFV), Rio Paranaíba, MG, Brasil.
}

\section{Resumo}

Introdução: a obesidade é um problema de saúde pública que está associada ao processo de ingestão dos alimentos. Objetivo: avaliar a mastigação e a saciedade das crianças de uma Escola Estadual. Métodos: avaliaram-se as crianças quanto à mastigação e à saciedade, mediante o consumo de maçã e biscoito com iogurte. Utilizou-se uma Escala Visual Analógica para avaliar a saciedade. Aplicou-se o Teste de Wilcoxon para avaliar, no mesmo sexo, o tempo de ingestão, a quantidade consumida e a saciedade; e o Teste de Mann Whitney para comparar entre os diferentes sexos e a mesma classificação do estado nutricional, sendo que ambos foram analisados a $5 \%$ de significância. Resultados: participaram do estudo 25 crianças eutróficas e 25 com excesso de peso. As meninas com excesso de peso consumiram a maçã em menor tempo $(p<0,05)$. Ao comparar as crianças de diferentes sexos e a mesma classificação do estado nutricional, os meninos apresentaram um menor tempo de consumo para ambos os alimentos, e aqueles com excesso de peso ingeriram uma maior quantidade $(\mathrm{p}<0,05)$. Não houve diferença estatística nos sexos, nos valores da Escala Visual Analógica para os alimentos avaliados, aos quais os meninos com excesso de peso atribuíram-se menores notas $(\mathrm{p}<0,05)$, quando se compararam os diferentes sexos e a mesma classificação do estado nutricional. Conclusão: as crianças com excesso de peso consumiram, em menor tempo, uma maior quantidade de alimentos, havendo uma menor saciedade. É importante a realização de atividades de educação nutricional com essas crianças, visando à correta ingestão de alimentos para a prevenção e o tratamento do excesso de peso.

Palavras-chave: Escala Visual Analógica. Alimentos. Obesidade.

\begin{abstract}
Introduction: obesity is a public health problem that is associated with the food intake process. Objective: to evaluate the chewing and satiety of the children of a State School. Methods: children were evaluated for chewing and satiety by eating apple and biscuit with yogurt. A Visual Analog Scale was used to evaluate satiety. The Wilcoxon Test was used to evaluate the time of ingestion, the amount consumed and the satiety; and the Mann Whitney Test to compare the different sexes and the same classification of the nutritional status, both of which were analyzed at $5 \%$ of significance. Results: 25 eutrophic children and 25 overweight children participated in the study. Overweight girls consumed the apple in less time ( $<<0.05$ ). When comparing children of different sexes and the same classification of nutritional status, the boys had a shorter consumption time for both foods, and those overweight ate a greater amount $(\mathrm{p}<0.05)$. There was no statistical difference in the sexes, from the Visual Analogue Scale values for the evaluated foods, in which overweight boys assigned lower scores $(\mathrm{p}<0.05)$ when comparing the different sexes and the same classification of the nutritional status. Conclusion: overweight children consumed a smaller amount of food in less time, with less satiety. It is important to carry out nutrition education activities with these children, aiming at the correct intake of food for the prevention and treatment of excess weight.
\end{abstract}

Key words: Visual Analogue Scale. Foods. Obesity.

\section{INTRODUÇÃO}

A obesidade é um problema de saúde pública que está associada ao surgimento das doenças crônicas não transmissíveis, como a hipertensão arterial, o diabetes mellitus, as doenças cardiovasculares e algumas neoplasias ${ }^{1}$. É preocupante a prevalência do excesso de peso em crianças e adolescentes, sugerindo que suas rotinas diárias sejam alteradas para que estes indivíduos apresentem um futuro mais saudável ${ }^{1}$.

A mastigação adequada é importante e consiste na primeira fase da deglutição. Ela pertence ao sistema estomatognático, cujo objetivo é quebrar o alimento em pequenas partículas para facilitar a deglutição e a digestão enzimática. No entanto, para que o processo seja efetivo, é importante que o indivíduo esteja com a postura ereta, que os dentes sejam íntegros, e que as articulações controlem os movimentos da mandíbula de forma correta² $^{2}$ A mastigação exerce influência direta no balanço energético, reduzindo a ingestão alimentar e alterando a digestão, no qual o aumento do número de ciclos mastigatórios promove a saciedade ${ }^{3}$.

As crianças devem estar atentas à forma e ao número de repetições que ocorrem na mastigação, pois quando ela é realizada de forma rápida e inadequada, pode ser um fator de risco para o excesso de peso, visto que a sinalização da saciedade cerebral não é realizada de forma instantânea, e o indivíduo poderá se alimentar excessivamente ${ }^{4}$.

O objetivo do estudo foi avaliar a mastigação e a saciedade das crianças de uma Escola Estadual de Rio Paranaíba, Minas Gerais, Brasil.

\section{MÉTODOS}

Trata-se de um estudo transversal, quantitativo que teve início

Correspondência: Martha Elisa Ferreira de Almeida. Universidade Federal de Viçosa, Campus Rio Paranaíba, Km 7, Zona Rural, Rio Paranaíba, MG, CEP: 38810-000. E-mail: martha.almeida@ufv.br. 
após a aprovação do Comitê de Ética e Pesquisa da Universidade Federal de Viçosa (UFV), parecer no. 1.203.181, assim como a assinatura e a entrega pelos cuidadores e crianças dos Termos de Consentimento Livre e Esclarecido e de Assentimento Livre e Esclarecido.

No segundo semestre de 2015, avaliaram-se 132 (53,23\%) crianças na faixa etária de 5 a 9 anos e 11 meses, matriculadas em uma Escola Estadual de Rio Paranaíba, Minas Gerais, Brasil. Para a seleção da amostra, realizou-se o pareamento dos indivíduos segundo o mesmo sexo, idade, turno matriculado, e classificação do estado nutricional. Ao selecionar uma menina de seis anos, com excesso de peso, para compor o seu par alocou-se uma menina eutrófica de seis anos, e assim, sucessivamente.

Para a realização das medidas antropométricas, encaminhou-se cada criança individualmente para uma sala, onde se aferiram o peso e a altura, com o auxílio de uma balança digital (marca Marte ${ }^{\circledR}$ com a escala $0,1 \mathrm{~kg}$, sendo a carga máxima de 150 $\mathrm{kg}$ ) e um estadiômetro (marca Alturexata ${ }^{\circledR}$ com a escala em milímetros). Para a obtenção do peso, as crianças estavam descalças e com o mínimo de vestimentas, e aferiu-se a estatura com o auxílio do estadiômetro, a qual apresentava uma posição ereta, com os pés juntos, mãos ao lado do corpo e cabeça posicionada em ângulo de $90^{\circ} \mathrm{com}$ o olhar fixo para o horizonte, sem fazer flexão ou estender a cabeça. Os dados de peso e de altura foram classificados nas curvas de crescimento expressas em percentis e escore-z dos índices IMC e Estatura para Idade (5-19 anos em meninos e meninas) de acordo com os parâmetros indicadores do estado nutricional ${ }^{5}$. A partir de tal análise, classificou-se as crianças como eutróficas ou com excesso de peso.

Na semana seguinte, avaliaram-se as crianças selecionadas quanto à mastigação e à saciedade, mediante o consumo de alguns alimentos. Elas foram encaminhadas individualmente para uma sala, onde receberam orientações para permanecerem sentadas em frente ao avaliador, de forma ereta e com o olhar para o horizonte de acordo com o plano de Frankfurt, e os pés totalmente apoiados ao chão ${ }^{6}$.

No primeiro dia de avaliação, entregou-se, individualmente para cada criança uma unidade pequena de maçã (previamente higienizada com solução clorada), e no segundo dia, ofereceramse $100 \mathrm{~mL}$ de iogurte sabor morango e três unidades de biscoito doce integral. Os alimentos foram escolhidos pela necessidade de mastigação, o que resultaria em um maior tempo para a sua ingestão, sendo que o iogurte foi adicionado para acompanhar a ingestão do biscoito.

$\mathrm{Na}$ pesagem direta dos alimentos, utilizou-se uma balança portátil (marca Plenna ${ }^{\circledR}$, com a capacidade máxima de $5 \mathrm{Kg}$, e a precisão $1 \mathrm{~g}$ ). Para determinar o resto da ingestão de cada criança, os alimentos deixados, no prato ou no copo descartável, foram pesados e subtraídos do peso do alimento distribuído. A avaliação ocorreu antes do oferecimento da alimentação escolar, no turno matutino (07:00 as 09:00 horas) e no turno vespertino (12:00 as $14: 00$ horas). As crianças receberam a orientação para mastigar e para engolir os alimentos da forma mais natural possível, e deveriam levantar a mão direita para sinalizar o início e o término da cronometragem do tempo de mastigação e da ingestão alimentar.

Durante a mastigação e a ingestão de alimentos, as crianças foram analisadas quanto às características da manutenção da cavidade oral. Para avaliar a saciedade dos alimentos ingeridos utilizou-se a Escala Visual Analógica ${ }^{7}$, na qual cada criança recebeu, após a finalização da cronometragem do tempo de ingestão, uma escala de $10 \mathrm{~cm}$ disposta horizontalmente em uma folha de papel ofício, cujo traço vertical localizado na extremidade esquerda do teste representava "pouco satisfeito(a)" e na extremidade direita "muito satisfeito(a)". Pediu-se aos participantes que assinalassem na escala, com um traço na vertical, a saciedade sentida no fim da ingestão dos alimentos de cada dia do estudo. A distância da extremidade esquerda até a marca assinalada pelo avaliado foi medida em centímetros para cada alimento-teste.

Os dados das características do estado de manutenção da cavidade oral durante a mastigação entre os dois grupos de crianças foram expressos como frequências absoluta e relativa. Utilizou-se o Teste de Wilcoxon para avaliar as médias dos tempos de ingestão e a quantidade ingerida dos alimentos; e entre as médias dos valores obtidos na Escala Visual Analógica. Para relacionar o IMC entre os sexos e a mesma classificação do estado nutricional, e as médias dos tempos de ingestão com a classificação do estado nutricional utilizou-se o Teste de MannWhitney. As análises foram realizadas no software Statistical Package for the Social Sciences (SPSS), versão 20.0, a 5\% de probabilidade.

\section{RESULTADOS}

Participaram do estudo 25 crianças eutróficas e 25 com excesso de peso, 32 meninas e 18 meninos (figura 1). Os valores médios de IMC não diferiram entre as crianças com a mesma classificação do estado nutricional $(p>0,05)$.

Figura 1. Média e desvio padrão do Índice de Massa Corporal das crianças, segundo o sexo e a classificação do estado nutricional. Rio Paranaíba - MG.

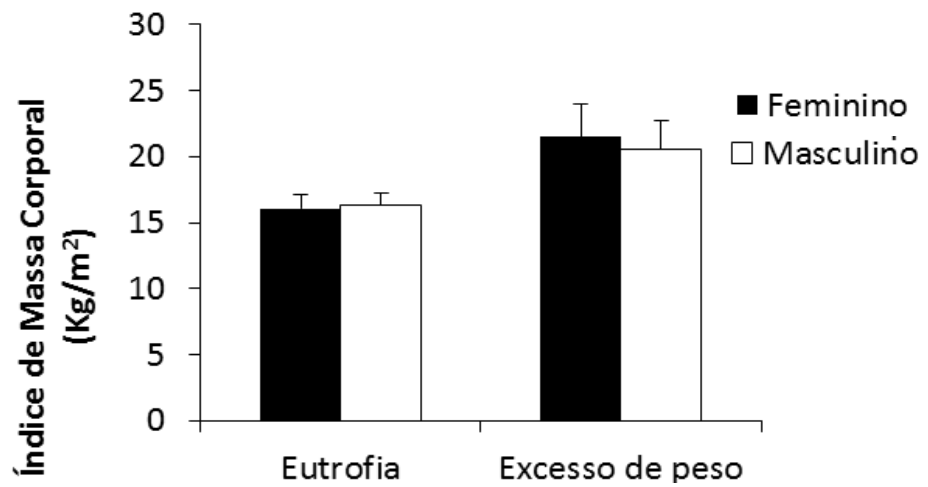


Quanto ao tempo de ingestão dos alimentos avaliados, houve diferença estatística $(p<0,05)$ apenas entre o sexo feminino para o consumo da maçã, e aquelas com excesso de peso consumiram em menor tempo (Tabela 1). Quando se avaliaram as crianças de diferentes sexos e a mesma classificação do estado nutricional, aqueles do sexo masculino (eutróficos e com excesso de peso) apresentaram um menor tempo de ingestão alimentar de todos os alimentos avaliados $(p<0,05)$.

Ao avaliar a quantidade consumida dos alimentos, não se observou diferença estatística no sexo feminino, embora os meninos com excesso de peso tenham consumido uma maior quantidade (Tabela 1).

Não houve diferença $(p>0,05)$ dos valores da Escala Visual Analógica para os alimentos avaliados quanto se comparou o sexo e a classificação do estado nutricional. Entretanto, quando se avaliam os diferentes sexos e o excesso de peso, os meninos atribuíram menores notas $(p<0,05)$ em tal escala (Tabela 1$)$, ressaltando que os meninos com excesso de peso consumiram uma maior quantidade dos alimentos em um menor tempo, e atribuíram menores notas na avaliação da saciedade.

Tabela 1. Valores médios e desvio padrão do tempo de consumo, quantidade consumida e da nota atribuída na Escala Visual Analógica dos alimentos avaliados, segundo o sexo e a classificação do estado nutricional. Rio Paranaíba - MG.

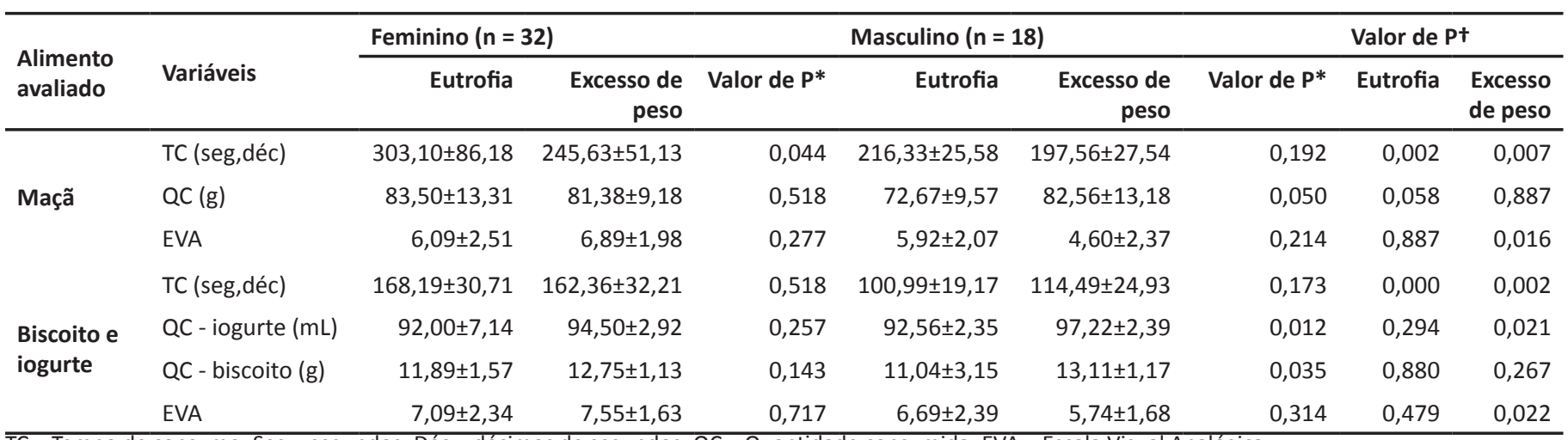

TC = Tempo de consumo; Seg = segundos; Déc = décimos de segundos; QC = Quantidade consumida; EVA = Escala Visual Analógica.

*Valor de P segundo o Teste de Wilcoxon quanto a classificação do estado nutricional e o sexo.

+Valor de P segundo o Teste de Mann-Whitney entre a mesma classificação e o sexo.

Ao consumirem a maçã, a maioria das crianças apresentou uma mordida frontal $(n=45)$ e um tipo mastigatório unilateral $(n=$ 34), independente da classificação do estado nutricional e do sexo (Tabela 2). Nenhuma criança avaliada apresentou mordida lateral ou partiu o alimento com as mãos. Todos os meninos com excesso de peso apresentaram a mordida frontal e o tipo mastigatório unilateral. A maior parte das crianças apresentou um ritmo mastigatório médio e lábios fechados, com a presença de ritmo rápido para duas crianças do sexo masculino e quatro do feminino. Não foram observados tosse, engasgos, alteração facial ou ruídos durante o consumo da maçã nas crianças, independente de sua classificação do estado nutricional.

Tabela 2. Frequência absoluta e relativa das crianças quanto o consumo da maçã, segundo o sexo e a classificação do estado nutricional. Rio Paranaíba - MG.

\begin{tabular}{llrrrr}
\hline & & \multicolumn{2}{c}{ Feminino } & \multicolumn{2}{c}{ Masculino } \\
\cline { 3 - 6 } & \multirow{2}{*}{ Item avaliado } & $\begin{array}{r}\text { Eutrofia } \\
\mathbf{N}(\%)\end{array}$ & $\begin{array}{r}\text { Excesso de peso } \\
\mathbf{N}(\%)\end{array}$ & $\begin{array}{r}\text { Eutrofia } \\
\mathbf{N}(\%)\end{array}$ & $\begin{array}{r}\text { Excesso de peso } \\
\mathbf{N}(\%)\end{array}$ \\
\hline \multirow{2}{*}{ Mordida } & Frontal & $14(87,50)$ & $14(87,50)$ & $8(88,89)$ & $9(100,00)$ \\
& Fronto-lateral & $2(12,50)$ & $2(12,50)$ & $1(11,11)$ & - \\
\multirow{2}{*}{ Tipo mastigatório } & Bilateral & $7(43,75)$ & $8(50,00)$ & $1(11,11)$ & - \\
& Unilateral & $9(56,25)$ & $8(50,00)$ & $8(88,89)$ & $9(100,00)$ \\
\multirow{2}{*}{ Ritmo } & Lento & $5(31,25)$ & $2(12,50)$ & $2(22,22)$ & - \\
& Médio & $11(68,75)$ & $10(62,50)$ & $6(66,67)$ & $8(88,89)$ \\
& Rápido & - & $4(25,00)$ & $1(11,11)$ & $1(11,11)$ \\
\hline \multirow{2}{*}{ Lábios } & Abertos & - & - & $1(11,11)$ & - \\
& Fechados & $16(100)$ & $14(87,50)$ & $8(88,89)$ & $7(77,78)$ \\
& Abertos e fechados & - & $2(12,50)$ & & - \\
\hline
\end{tabular}


$\mathrm{Na}$ ingestão do biscoito e do iogurte, independente da classificação do estado nutricional, a maioria das crianças apresentou o tipo de mordida frontal ( $n=44)$, e nenhuma partiu o biscoito com as mãos (Tabela 3). Todos os meninos avaliados, independente da classificação do estado nutricional, apresentaram tipo mastigatório unilateral e lábios fechados, com um ritmo médio na maioria das crianças. Não houve tosse, engasgos, alteração facial ou ruídos durante o consumo desses alimentos.

Tabela 3. Frequência absoluta e relativa das crianças quanto o consumo do biscoito e do iogurte, segundo o sexo e a classificação do estado nutricional. Rio Paranaíba - MG.

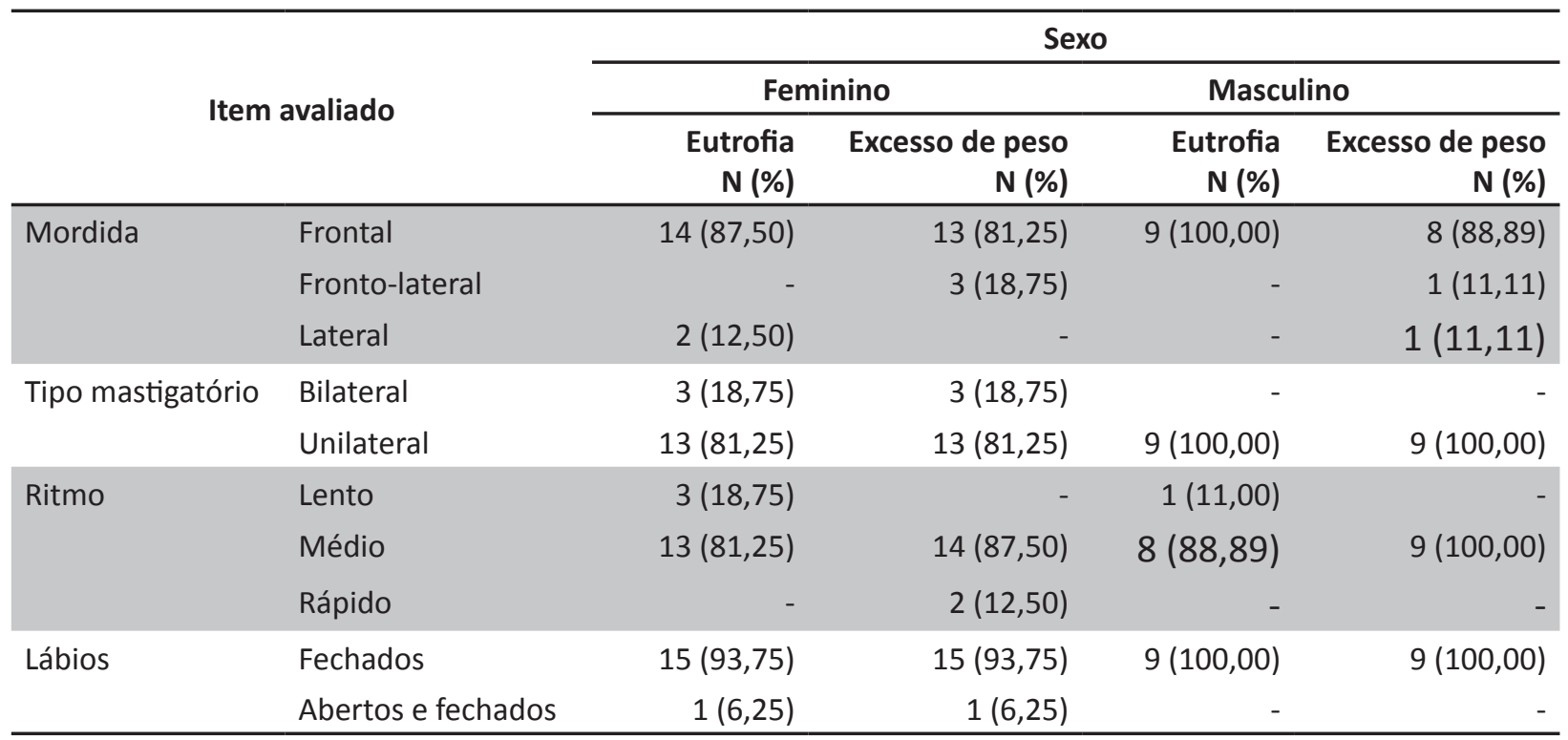

\section{DISCUSSÃO}

Algumas crianças avaliadas neste estudo apresentaram IMC maior que $20 \mathrm{~kg} / \mathrm{m}^{2}$. Tem sido crescente o número de indivíduos de 5 a 9 anos de idade com excesso de peso, sendo que, na última Pesquisa de Orçamentos Familiares (2008/2009), $34,80 \%$ do sexo masculino e $32,00 \%$ do feminino apresentaram tal classificação do estado nutricional ${ }^{8}$. Segundo Costa et al. ${ }^{1}$ a ocorrência excesso de peso na infância é preocupante, visto que poderá gerar uma grande repercussão na vida adulta com uma redução da qualidade de vida, presença de baixa estima, gastos financeiros elevados na saúde pública, além do surgimento de patologia como diabetes mellitus tipo 2, hipertensão arterial, doenças cardiovasculares e alguns tipos de cânceres, assim como a permanência do excesso de peso (sobrepeso ou obesidade) quando adultos.

As crianças com excesso de peso apresentaram um menor tempo de ingestão dos alimentos, assim como descrito por Gonçalves e Chehter ${ }^{4}$ que identificaram que os indivíduos com excesso de peso apresentam um ritmo mastigatório mais rápido. É primordial que os indivíduos mastiguem várias vezes os alimentos e de forma lenta, para facilitar a digestão e evitar a liberação tardia da leptina, um dos hormônios relacionados com a saciedade ${ }^{9}$.

A mordida frontal para a maçã e o biscoito foi identificada entre a maioria das crianças, e nenhuma partiu os alimentos com as mãos. $\mathrm{O}$ ato da mastigação promove uma maior maceração do alimento que auxilia no processo da digestão ao aumentar a superfície de contato para a atuação das enzimas digestivas ${ }^{6,10}$. Apolinário et al. ${ }^{11}$ identificaram que $46,70 \%$ dos avaliados tinham uma mordida frontal, e $46,70 \%$ partiram o alimento com as mãos, sendo sugerido por Junqueira ${ }^{12}$ que os indivíduos realizem a mordida frontal do alimento. Entretanto, Cunha et al. ${ }^{13}$ recomendam que a mastigação deve ser bilateral para impedir alterações no sistema estomatognático, pois, assim, irá ocorrer a distribuição da força mastigatória, não alongando, de forma excessiva, o tônus de nenhum dos lados da mandíbula, e ocorra a intercalação dos períodos de mastigação, visando o equilíbrio muscular e funcional.

Independente da classificação do estado nutricional, a maioria das crianças apresentou uma mastigação unilateral, assim como identificado por Garcia et al. ${ }^{14}$ com escolares de 7 a 12 anos de idade, os quais $65,50 \%$ apresentavam uma propensão à escolha do lado direito. Entretanto, Souza e Guedes ${ }^{15}$ identificaram que $68,00 \%$ das crianças e adolescentes obesos apresentaram mastigação bilateral e uma menor eficiência da deglutição.

A maioria das crianças apresentou ritmo mastigatório médio e lábios fechados, independente da classificação do estado nutricional, para os alimentos testados. Gonçalves e Chehter ${ }^{4}$ ao avaliarem o consumo de pão de queijo por adultos eutróficos e com obesidade mórbida, observaram uma predominância do ritmo mastigatório rápido. O ritmo acelerado impede 
que os sabores sejam identificados nas papilas gustativas, e consequentemente, os estímulos de saciedade serão transmitidos lentamente, o que poderá ocasionar o consumo rápido e excessivo de alimentos, principalmente naqueles indivíduos com excesso de peso ${ }^{4}$.

Os alimentos podem aumentar a saciedade a partir da estimulação oral e sensorial, uma vez que sua exposição, por mais tempo na cavidade oral, diminui a ingestão de energia e o volume gástrico final, destacando a importância da mastigação lenta como forma de maior controle da saciedade e da ingestão calórica ${ }^{16}$.

Zhu et al. ${ }^{17}$ quando avaliaram a saciedade de indivíduos adultos, ofereceram inicialmente pedaços de pizzas, e três horas depois, outra refeição à base de massas (espaguete com vários tipos de molhos) observaram que os participantes, que apresentaram mais ciclos mastigatórios durante o consumo da pizza, obtiveram uma maior saciedade, e um menor volume ingerido na refeição seguinte, uma maior concentração plasmática de colecistoquinina e um menor valor sérico de grelina, insulina e glicose.

A presença dos lábios fechados durante a mastigação dos alimentos foi identificada na maioria das crianças, assim como observado por Apolinário et al. ${ }^{11}$ que observaram que $86,70 \%$ dos acadêmicos do Curso de Nutrição permaneceram com os lábios cerrados durante a mastigação do pão de sal. Duarte e Ferreira ${ }^{18}$ destacam que é importante que os lábios se mantenham fechados durante a ingestão alimentar, pois assim irá auxiliar na pressão intraoral para o lançamento correto do bolo alimentar até a faringe, evitando sua saída pela boca.

Miguel-Kergoat ${ }^{19}$ ao realizarem uma revisão sistemática sobre a mastigação e o apetite identificaram que a mastigação diminui a fome e ingestão de alimentos, possivelmente pela resposta dos hormônios relacionados à saciedade. Entre os estudos avaliados, identificou-se que a mastigação correta reduziu sua ingestão; há uma relação da mastigação e a saciedade, demonstrando que o aumento do número de mastigações por mordida aumentou a liberação da colecistocinina e do peptídeo inibidor gástrico, e diminuiu a secreção de grelina.

O bolo alimentar deve estar presente na cavidade oral até o momento de sua deglutição, pois caso ocorra tosse e engasgos durante a mastigação e antes da deglutição, pode ser um indicativo da saída do mesmo em direção a faringe, cuja dificuldade de manter o bolo alimentar na cavidade oral se deve as alterações motoras ou sensitivas no sistema digestório ${ }^{20}$.
Não houve a presença de ruídos durante o consumo dos alimentos avaliados neste estudo, diferindo daquele realizado com acadêmicos do Curso de Nutrição, em que 6,70\% apresentaram ruídos durante o consumo do pão de sal ${ }^{10}$.

A mastigação pode auxiliar no processo de digestão, uma vez que a amilase salivar age na região bucal. Os alimentos que apresentam uma grande superfície de contato gastam um maior tempo de digestão quando comparados àqueles que foram mais triturados, evitando, assim, sua longa permanência no estômago e no intestino delgado. Quando o alimento é mastigado, inadequadamente e de forma insuficiente, pode haver a presença de grandes volumes de ar no estômago, necessitando de um maior trabalho desse órgão para realizar a mistura do bolo alimentar com os sucos gástricos e as enzimas que irão digerir as proteínas ${ }^{4}$. Assim, a população dever ser orientada quanto à mastigação, visto que o ritmo mastigatório pode influenciar negativamente na saciedade, e consequentemente contribuir para o excesso de peso ${ }^{11}$.

Como limitação deste estudo, destaca-se o reduzido número de crianças avaliadas, sugerindo que outras pesquisas sejam realizadas com uma maior amostra de crianças, bem como adolescentes.

\section{CONCLUSÃO}

O tempo de mastigação apresentou uma associação com a classificação do estado nutricional das crianças, uma vez que os meninos com excesso de peso consumiram uma maior quantidade dos alimentos em um menor tempo, e foram atribuídas menores notas na avaliação da saciedade. O tipo mastigatório unilateral foi predominante em todas as crianças, e, na maioria dos avaliados, houve a presença da mordida frontal e um ritmo médio.

Torna-se necessária a realização de atividades de educação nutricional com todas as crianças, principalmente, aquelas com excesso de peso, uma vez que o tempo de ingestão dos alimentos, como também as características da mastigação podem contribuir para uma baixa saciedade, e propiciar o ganho de peso.

\section{AGRADECIMENTOS}

À direção da Escola Estadual avaliada, aos alunos participantes do estudo, e todos aqueles que contribuíram para sua realização.

\section{REFERÊNCIAS}

1. Costa KCM, Ciampo LAD, Silva PS, Lima JC, Martins WP, Nogueira-de-Almeida CA. Marcadores ultrassonográficos de risco cardiovascular em crianças obesas. Rev Paul Pediatr. 2018 Jun; 36(2): 171-175. http://dx.doi.org/10.1590/19840462/;2018;36;2;00016.

2. Mélo TMA, Carvalho CC, Calvacanti AS, Dourado MG Filho, Pinheiro, PF Junior, Silva HJ. Estudo das relações entre mastigação e postura de cabeça e pescoço
- revisão sistemática. Rev CEFAC. 2012 Abr; 14(2): 327-332. doi: org/10.1590/ S1516-18462011005000125.

3. Holis JH. The effect of mastication on food intake, satiety and body weight. Physiol Behav. 2018 Sep; 193: 242-245. doi: 10.1016/j.physbeh.2018.04.027.

4. Gonçalves RFM, Chehter EZ. Perfil mastigatório de obesos mórbidos 
submetidos à gastroplastia. Rev CEFAC. 2012 Maio-Jun; 14(3): 489-497. doi: http://dx.doi.org/10.1590/S1516-18462011005000137.

5. World Healt Organization. Growth reference data for 5-19 years [Internet] Geneva: WHO; 2018[cited 2018 Set 14]. Available from: http://www.who.int/ growthref/en/.

6. Whitaker ME, Trindade Júnior AS, Genaro KF. Proposta de protocolo de avaliação clínica da função mastigatória. Rev CEFAC. 2009; 11(Supl 3): 311-323. doi: 10.1590/S1516-18462009005000030.

7. Corrêa LL, Platt MW, Moreira RO, Faria Júnior R, Godoy-Matos AF, Meirelles RMR, et al. Avaliação do efeito da sibutramina sobre a saciedade por escala visual analógica em adolescentes obesos. Arq Bras Endocrinol Metab. 2005 Abr; 49(2): 286-290. doi: org/10.1590/SOOO4-27302005000200016.

8. Instituto Brasileiro de Geografia e Estatística. Pesquisa de Orçamentos Familiares - Antropometria e Estado Nutricional de Crianças, Adolescentes e Adultos no Brasil. Rio de Janeiro: IBGE; 2010. p. 58-59.

9. Vieira ACC, Santos VA, Santos CPF, Costa MFF, Ferreira MLB. Perfil do Sistema Estomatognático nos portadores de esclerose múltipla, atendidos no Centro de Referência do Hospital da Restauração Recife - PE - Brasil. Rev Neurobiologia. 2010 Jan-Mar; 73(1): 85-91.

10. Felício CM, Couto GA, Ferreira CLP, Mestriner Junior W. Confiabilidade da eficiência mastigatória com beads e correlação com a atividade muscular. Pró-Fono R Atual Cient. 2008 Out-Dez; 20(4): 225-230. doi:10.1590/S010456872008000400004 .

11. Apolinário RMC, Moraes RB, Motta AR. Mastigação e dietas alimentares para redução do peso. Rev CEFAC. 2008; 10(2): 191-199. doi: org/10.1590/ S1516-18462008000200008.

12. Junqueira P. Avaliação miofuncional. In: Marquesan, IQ. Fundamentos em fonoaudiologia: aspectos clínicos da motricidade oral. Rio de Janeiro:
Guanabara Koogan; 1998. cap. 3, p. 13-21, 1998.

13. Cunha DA, Silva GAP, Motta MEFA, Lima CR, Silva HJ. A respiração oral em crianças e suas repercussões na classificação nutricional. Rev CEFAC. 2007 JanMar; 9(1): 47-54. doi: org/10.1590/S1516-18462007000100007.

14. Garcia DGB, Benevides SD, Araujo RP, Ribeiro CO, Mello SMF. Mastigação habitual e atividade eletromiográfica dos músculos masseter e temporal em escolares de 7 a 12 anos. Rev CEFAC. 2014 Dez; 16(6): 1928-1935. doi: org/10.1590/1982-0216201412413.

15. Souza NC, Guedes ZCF. Mastigação e deglutição de crianças e adolescentes obesos. Rev CEFAC. 2016 Dez; 18(6): 1340-1347. doi: dx.doi.org/10.1590/19820216201618617015.

16. Wijlens AGM, Erkner A, Alexander E, Mars M, Smeets PAM, Graff C. Effects of oral and gastric stimulation on appetite and energy intake. Obesity. 2012 Nov; 20(11): 2226-2232. doi:10.1038/oby.2012.131.

17. Zhu $\mathrm{Y}, \mathrm{Hsu} \mathrm{WH}$, Hollis $\mathrm{JH}$. Increasing the number of masticatory cycles is associated with reduced appetite and altered postprandial plasma concentrations of gut hormones, insulin and glucose. Br J Nutr. 2013 Jul; 110(2): 384-390. doi: 10.1017/S0007114512005053.

18. Duarte LIM, Ferreira LP. Respiração e mastigação: estudo comparativo. R Dental Press Ortodon Ortop Facial. 2003; 8(4): 79-87.

19. Miguel-Kergoat S, Azais-Braesco V, Burton-Freeman B, Hetherington MM. Effects of chewing on appetite, food intake and gut hormones: a systematic review and meta-analysis. Physiol Behav. 2015 Nov; 1(151): 88-96. doi: 10.1016/j.physbeh.2015.07.017.

20. Cattoni DM. Alterações da mastigação e deglutição. In: Ferreira LP, BefiLopes DM, Limongi SCO. Tratado de fonoaudiologia. São Paulo: Roca; 2004. cap. 24. p. $277-291$

\section{Como citar este artigo/How to cite this article:}

Santos BV, Almeida MEF. Análise da mastigação e da saciedade em escolares. J Health Biol Sci. 2019 Jan-Mar; 7(1):47-52. 